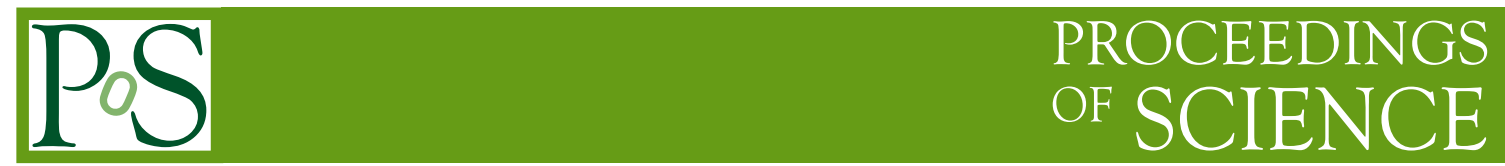

\title{
Indirect Dark Matter Searches: Status and Prospects
}

\section{Eric Charles*}

Kavli Institute for Particle Astrophysics and Cosmology, SLAC National Accelerator Laboratory, Stanford University, Stanford, CA 94305, USA

E-mail: echarleseslac.stanford.edu

I present of summary of the current status of searches for signals from dark matter interactions in astrophysical data. I also discuss the prospects for such searches in the next few years.

2nd World Summit: Exploring the Dark Side of the Universe 25-29 June, 2018 - EDSU2018

University of Antilles, Pointe-à-Pitre, Guadeloupe, France

${ }^{*}$ Speaker. 


\section{Introduction}

Overwhelming evidence indicates that the matter in the Universe can not only consist of particles in the Standard Model of particle physics. In particular, measurements of galactic rotation curves (see e.g., [1]) and galaxy cluster dynamics (see e.g., [2]), precision measurements of the cosmic microwave background (see e.g., [3, 4]), observations of the primordial abundances of heavy isotopes produced by Big Bang nucleosynthesis (see e.g., [5]), all point to a substantial fraction of the Universe's energy density being in a form of matter that does not interact signifcantly with the Standard Model particles. Numerical simulations of large-scale structure also support this conclusion; in fact, such simulations require non-relativistic dark matter (DM) in order to be consistent with observations (see e.g., [6] and references therein).

The observational evidence implies that DM is non-relativistic (i.e., "cold") during the formation of large-scale structure (see e.g., [3, 4]), and does not have large scattering cross sections with either itself (see e.g., [7]) or Standard Model particles (see e.g., [8]). No particle in the Standard Model meets the requirements. We have no other solid experimental or theoretical understanding of the fundamental nature of DM.

Two favored candidates for the DM particle are weakly interactive massive particles (WIMPs, with masses in the $\mathrm{GeV}$ to $\mathrm{TeV}$ range) and axions/ axion-like particles (ALPs, whose masses very poorly constrained and could range anywhere from $10^{-10}$ to $10^{9} \mathrm{eV}$. Both types of candidates could be detected via signatures in astrophysical data (a.k.a., "indirect-detection" searches).

\section{Status of Current Indirect Detection Dark Matter Searchs}

Indirect dectection WIMP searches consist of looking for anomolous $\gamma$-ray signals correlated with regions or astrophysical structures known to host large amounts of DM. The analysis targets are identified from a combination of analysis of kinematic data and study of numerical simulations.

Tab. 1 (taken from Ref. [9]) summarizes the targets for WIMP searches, including the astrophysical "J-factor", which is proportional to the expected signal magnitude for as given DM interaction rate. The differences between the targets are pronounced enough to warrant significant modifications in the search techniques. For example, searches targeting known dark-matter dominated Milky Way satellites are very similar to blind searches for point-like emission performed when creating catalogs of $\gamma$ ray point sources; on the other hand, extracting a isotropic signal from DM halos of galaxies at cosmological distances requires very detailed modeling of both the Galactic foreground emission and the contributions of unresolved sources.

\begin{tabular}{cccc}
\hline \hline Target & Distance $(\mathrm{kpc})$ & $\mathrm{J}$ factor $\left(\mathrm{GeV}^{2} \mathrm{~cm}^{-5}\right)$ & Angular Extent $\left(^{\circ}\right)$ \\
\hline Galactic center / halo & 8.5 & $3 \times 10^{22}$ to $5 \times 10^{23}$ & $>10$ \\
Known Milky Way satellites & 25 to 300 & $3 \times 10^{17}$ to $3 \times 10^{19}$ & $<0.5$ \\
Dark satellites & up to 300 & up to $3 \times 10^{19}$ & $<0.5$ \\
Galaxy Clusters & $>5 \times 10^{4}$ & up to $1 \times 10^{18}$ & up to $\sim 3$ \\
Cosmological DM & $>10^{6}$ & - & Isotropic \\
\hline \hline
\end{tabular}

Table 1: Summary table of DM search targets. 
Because of differences in the datasets, DM profiles, and background modeling, these results should be taken as representative and absolute comparisons should be interpreted with caution. Details about the scenarios considered (e.g., the DM distribution) for each of the targets are provided in Table 2 of Ref. [9].

Currently, the deepest limits come from searches targeting the dwarf spheroidal Milky Way sattelite galaxies, and these limits are in tension with results that interpret an excess of $\gamma$ rays for the Galactic center as a possible DM signal.

Axions and ALPs can produced signatures in astrophysical data because of their couplings to photons. ALP-photon interactions can produce charateristic spectral features in $\gamma$-ray data. Additionally, ALP-photon mixing can provide a mechanism by which the attenuation of high-energy $\gamma$ rays is significanlty reduced. This could allow us to observe $\gamma$ rays produced in very dense environments or at very great distances.

\section{Prospects for upcoming Indirect Detection Dark Matter Searchs}

Ref. [9] made a detailed analysis of the sensitivity considerations for DM WIMP searches and derived these broad conclusions:

1. Searches targeting objects with substantial astrophysical backgrounds, such as the Galactic center, large Galaxy clusters, and the LMC are systematics limited up to high energies (e.g., $m_{\chi} \lesssim 10 \mathrm{TeV}$ for the Galactic center, $m_{\chi} \lesssim 1 \mathrm{TeV}$ for the LMC).

2. Searches for spectral lines from the Galactic halo benefit from additional discriminating power in the spectral domain, but are still systematics limited at low energies (e.g., $m_{\chi} \lesssim$ $100 \mathrm{GeV})$.

3. Searches targeting relatively small objects at high Galactic latitudes, in particular the dSphs, are background limited at lower energies, and signal limited at higher energies. For the combined analysis of dSphs the cross-over point comes at $m_{\chi} \sim 500 \mathrm{GeV}$.

In short, prospects for the most notable improvements to our senstivitty come from additional data for searches targeting the dSphs for higher DM masses $\left(m_{\chi} \gtrsim 100 \mathrm{GeV}\right)$ and from the expected discovery of additional high $J$ value targets.

Furthermore, searches targeting the dSphs have smaller modeling uncertainties and more robust determinations of the astrophysical $J$ factors than searches targeting other objects. Additionally these searches have among the best sensitivities across much of the relevant energy band. Overall these considerations suggest that, looking forward, searches targeting the dSphs will continue to have the best sensitivity. This can clearly be seen in Fig. 1.

The current limits on the ALP parameters are summarized in Fig. 2 (red shaded regions). The search for $\gamma$ rays from axion or ALP decays produced in neutron star interiors lead to the limits labeled "ALPs from NS" (gray hatched region, assuming the model-dependent factor $\mathscr{N}=$ 1) [10]. The limits constrain the QCD axion mass (black line and yellow band in Fig. 2) to be below $7.9 \times 10^{-2} \mathrm{eV}$. It should be noted that these limits not only depend on the axion and ALP coupling to photons but also on the axion and ALP production through nucleon-nucleon Bremsstrahlung. 


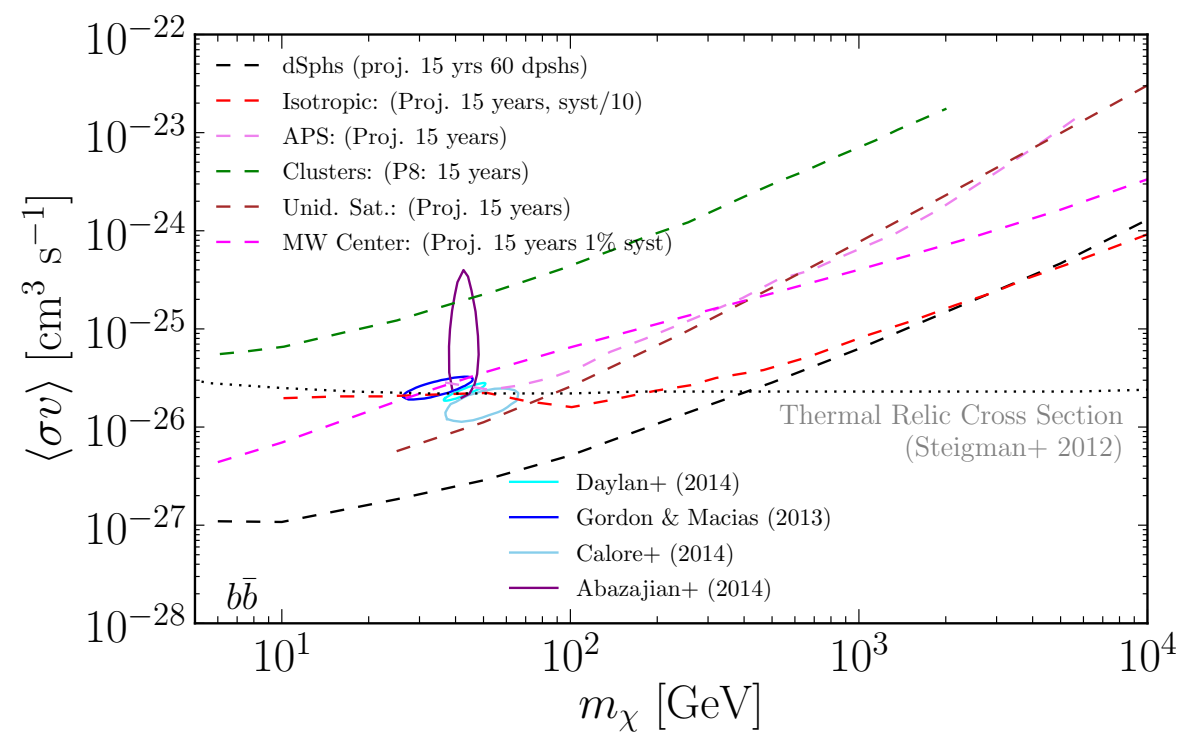

Figure 1: Comparison of projected LAT limits for 15 years of data for the search methods described in. Favored contours for several Galactic center analyses are also included for comparison.

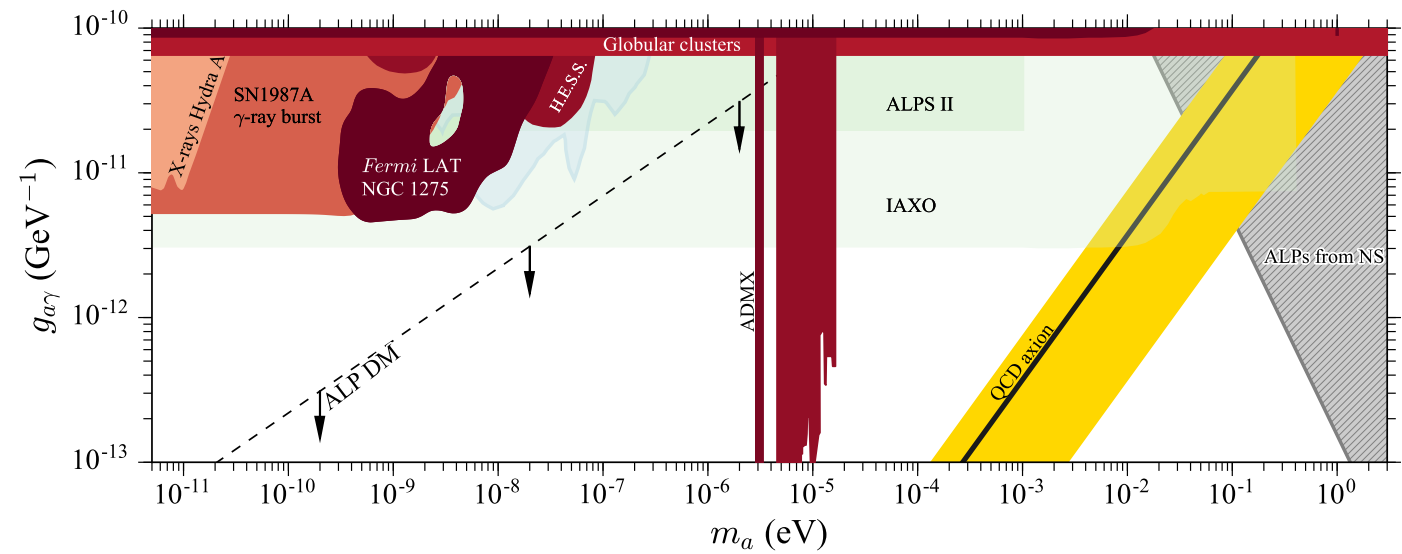

Figure 2: Current status of the limits imposed on the ALP parameter space by different experiments and targets. Limits derived with LAT observations are shown as dark red (NGC 1275 in the Perseus galaxy cluster [11]) and gray hatched (neutron stars [10]) regions. Limits from other experiments are shown in red. The parameter space where ALPs could explain a low $\gamma$-ray opacity is shown in light blue. The parameter space where ALPs could explain hints for a low $\gamma$-ray opacity are shown in blue. Sensitivity estimates for future laboratory experiments are shown in green. The QCD axion line is shown in yellow. ALP parameters below the dashed line could account for all the DM. See also Refs. [12, 13, 14, 15, 16, 17] and references therein. 


\section{References}

[1] V. Rubin, N. Thonnard and W.K. Ford Jr. Rotational properties of 21 SC galaxies with a large range of luminosities and radii, from NGC $4605 / R=4 \mathrm{kpc} /$ to $U G C 2885 / R=122 \mathrm{kpc} / \mathrm{Ap}$. J. (1980)

[2] F. Zwicky Die Rotverschiebung von extragalaktischen Nebeln, Helv.Phys.Acta (1933)

[3] P. A. R. Ade et al. Planck 2013 results. XVI. Cosmological parameters, A.A.P. (2014)

[4] P. A. R. Ade et al. Planck 2015 results. XIII. Cosmological parameters, A.A.P. (2015)

[5] K. A. Olive TASI lectures on dark matter astro-ph/0301505 (2003)

[6] C. S. Freck and S. D. M. White Dark matter evidence, particle physics candidates and detection methods Annalen der Physik (2012)

[7] M. Markevitch et al. Direct constraints on the dark matter self-interaction cross-section from the merging galaxy cluster 1E0657-56 Ap. J. (2004)

[8] D. S. Akerib et al. First results from the LUX dark matter experiment at the Sanford Underground Research Facility Phys. Rev. Lett. (2014)

[9] E. Charles et al. Sensitivity projections for dark matter searches with the Fermi Large Area Telescope Physics Reports. (2016)

[10] B. Berenji, J. Gaskins, M. Meyer Constraints on axions and axionlike particles from Fermi Large Area Telescope observations of neutron stars Phys. Rev. D (2016)

[11] M. Ajello, M. et al. Search for Spectral Irregularities due to Photon-Axionlike-Particle Oscillations With the Fermi Large Area Telescope Phys. Rev. Lett. (2016)

[12] R. Bähre et al. Any light particle search II - Technical Design Report Journal of Instrumentation. (2013)

[13] A. Payez et al. Revisiting the SN1987A gamma-ray limit on ultralight axion-like particles J. C. A. P. (2015)

[14] A. Abramowski et al. Constraints on axionlike particles with H.E.S.S. from the irregularity of the PKS 2155-304 energy spectrum Phys. Rev. D (2013)

[15] M. Meyer, D. Horns and M. Raue First lower limits on the photon-axion-like particle coupling from very high energy gamma-ray observations Phys. Rev. D (2013)

[16] M. Meyer and J. Conrad Sensitivity of the Cherenkov Telescope Array to the detection of axion-like particles at high gamma-ray opacities J. C. A. P. (2015)

[17] R. Essig et al. Dark Sectors and New, Light, Weakly-Coupled Particles ArXiv e-prints (2013) 